\title{
MemoGinga: Jogo Interativo para TV Digital como Exercício Cognitivo para Idosos
}

\author{
Luiz F. Matos ${ }^{1}$, Mayara H. Santos ${ }^{1}$, Yolanda Boechat ${ }^{2}$ \\ Glauco Amorim $^{3}$, Débora C. Muchaluat-Saade ${ }^{1}$ \\ ${ }^{1}$ Laboratório MídiaCom - Universidade Federal Fluminense \\ ${ }^{2}$ CRASI - Centro de Referência em Assistência à Saúde do Idoso \\ Universidade Federal Fluminense \\ Niterói, RJ \\ ${ }^{3}$ CEFET/RJ - Rio de Janeiro, RJ \\ \{lfmatosmelo, mayarahelena, debora\}@midiacom.uff.br \\ yolanda.boechat@gmail.com, gamorim@eic.cefet-rj.br
}

\begin{abstract}
The recent diffusion of the electronic games has been awakening the interest of researchers which investigate the relationship between the use of games and the increase of human cognitive performance. Starting with that interest, the application of electronic games in the increase of the cognitive performance of the elderly is being explored. In Brazil, access to TV is very common and exists in the homes of every social classes. Thus, electronic games available through the Brazilian Digital TV System have potential to reach great part of the elderly population in the country. The present paper proposes and evaluates the MemoGinga, a short-span memory test program, aimed firstly at the elderly public and implemented in the Ging a middleware for interactive digital TV. MemoGinga was evaluated with elderly users from CRASI - Center of Reference in Assistance to Elderly Health.
\end{abstract}

Resumo. A recente difusão dos jogos eletrônicos tem despertado o interesse de pesquisadores que investigam as relações entre o uso de jogos e o aumento do desempenho cognitivo humano. A partir desse interesse, o uso de jogos eletrônicos para melhora das funções cognitivas de idosos tem sido cada vez mais explorado. No Brasil, o acesso à TV é muito comum e presente nos lares de todas as classes sociais. Desta forma, jogos eletrônicos disponíveis através do Sistema Brasileiro de TV Digital tem potencial para atingir grande parte da população idosa no país. O presente artigo propõe e avalia o MemoGinga, um teste de memória recente, direcionado principalmente ao público idoso e implementado no middleware Ginga para TV digital interativa. MemoGinga foi avaliado com usuários idosos atendidos pelo CRASI - Centro de Referência em Assistência à Saúde do Idoso.

Palavras-chave: MemoGinga, exercício cognitivo, jogos sérios em saúde, Ginga, NCL, TV Digital.

\section{Introdução}

A difusão dos games e das plataformas de entretenimento tem atraído a atenção de pesquisadores, especialmente aqueles que estudam o impacto de seu uso nas funções cerebrais 
humanas. Um grande número de pesquisas aponta correlações entre o uso dos jogos eletrônicos e a melhora de faculdades mentais como memória [Oei and Patterson 2013], funções executivas e velocidade de processamento de informações [Nouchi et al. 2012]. Entretanto, esse campo de estudo ainda requer maior exploração.

A necessidade de pesquisas sobre o tema e o consequente desenvolvimento e estudo dos jogos cognitivos e seu impacto no desempenho mental dos jogadores estabelecem demandas relacionadas à concepção e distribuição dessas ferramentas. Em relação à concepção, determina-se que os jogos cognitivos devam ter funcionamento suficientemente simples para propiciar o rápido aprendizado de modo que não causem frustrações aos usuários [Ishihara et al. 2012]. Já abordando sua distribuição, os jogos devem ser de acesso amplo e facilitado para incentivar seu uso continuado.

A maioria dos jogos cognitivos disponíveis na Internet são desenvolvidos, principalmente, em duas plataformas: desktop ou mobile. Para que o jogo seja instalado e efetivamente utilizado, pressupõe-se que o usuário tenha uma habilidade tecnológica que não pode ser ignorada quando trata-se do público idoso. Além disso, uma grande parte dos sites que disponibilizam esses jogos estão escritos no idioma inglês, o que é outro fator que limita o acesso daqueles que não possuem familiaridade com a língua inglesa. Outro ponto que pode ser um empecilho para que idosos possam utilizar os jogos cognitivos é o tamanho da tela do dispositivo exibidor. Quanto menor a tela, mais difícil será identificar e efetuar as tarefas requisitadas pelo jogo.

No Brasil, o acesso à TV é muito comum e presente nos lares de todas as classes sociais. Desta forma, jogos eletrônicos disponíveis através do Sistema de TV Digital Brasileiro tem potencial para atingir grande parte da população idosa no país. Este artigo propõe e avalia o MemoGinga, um exercício cognitivo desenvolvido para o middleware Ginga [NBR 15606-2:2011 2011] do Sistema Brasileiro de TV digital (SBTVD). Uma versão inicial do jogo foi proposta em [Matos et al. 2018]. A versão apresentada neste artigo apresenta diferentes níveis de dificuldade e foi avaliada com um número maior de usuários.

O MemoGinga foi desenvolvido com a finalidade de estimular as funções cognitivas de idosos. Possui funcionamento intuitivo consistindo na exibição de figuras geométricas ao jogador e este deve dizer se elas são iguais ou diferentes das figuras exibidas anteriormente. A introdução do jogo, explicando as regras e seu objetivo, é feita através de um áudio e a língua escolhida para realização do mesmo é o português. A implementação para o sistema de TV digital brasileiro visa propiciar a difusão de acesso ao programa entre o público idoso em razão da popularidade deste meio de comunicação no Brasil [Governo Brasileiro 2014]. Além disso, como o tamanho das telas de TV são bem superiores se comparados a smartphones e monitores de computadores, espera-se que o usuário apresente um maior conforto ao identificar e realizar as tarefas requisitadas pelo jogo.

O restante do texto está estruturado da seguinte forma. Na Seção 2, são abordados trabalhos relacionados ao MemoGinga. A Seção 3 discute a relação entre jogos eletrônicos e cognição. A Seção 4 apresenta a proposta do MemoGinga e suas características de implementação. A avaliação do jogo é apresentada na Seção 5. Finalmente, a Seção 6 conclui o artigo com considerações finais e trabalhos futuros. 


\section{Trabalhos Relacionados}

Entre outros trabalhos que usam os jogos eletrônicos como forma de exercício cognitivo, destacam-se as plataformas "Lumosity"[Lumos Labs, Inc 2014], "Brain HQ"[Posit Science 2016] e "Fit Brains"[Rosetta Stone 2018].

Lumosity [Lumos Labs, Inc 2014] é uma ferramenta online onde os participantes podem treinar suas habilidades cognitivas essenciais. Diversas áreas são combinadas para criar treinamentos cognitivos. A plataforma inicia com alguns testes para criar um baseline e, após essa avaliação, indica testes a serem realizados. O usuário pode personalizar o treinamento. Os jogos, são divididos em velocidade, memória, atenção, flexibilidade e solução de problemas. Entretanto, para acessar a grande maioria dos jogos é necessário pagar por um plano. A ferramenta é disponibilizada nas plataformas web e mobile, o que exige dos usuários um conhecimento mínimo sobre essas plataformas para que se possa usufruir de todos os benefícios. Essas habilidades não são, necessariamente, constatadas no público alvo deste trabalho. Portanto, apesar de ser uma plataforma rica em funcionalidades pode não ser adequada ao público idoso.

Brain $H Q$ [Posit Science 2016] é uma ferramenta muito parecida com Lumosity. As categorias dos jogos são divididas em: atenção, cérebro, memória, socialização, inteligência e orientação. A interface da ferramenta propõe metas semanais que podem personalizadas e o usuário pode acompanhar se as metas foram ou não alcançadas através de gráficos que mostram atividades praticadas nos dias daquela semana. Para acessar todo o conteúdo da plataforma é necessário pagar por um plano, assim como o Lumosity. As observações sobre habilidades com tecnologia feitas anteriormente também se mantêm nessa plataforma.

Outra plataforma observada foi a Fit Brains [Rosetta Stone 2018]. De acordo com os autores, essa plataforma disponibiliza um programa de treinamento cerebral que mistura diversão com treinamento, pois foi projetada por especialistas em jogos com contribuições de neurocientistas. O programa fornece uma gama de jogos que envolvem várias áreas do cérebro como: memória, foco, velocidade de pensamento, lógica e resolução de problemas, reconhecimento espacial. Além das habilidades cognitivas, é possível treinar outras habilidades como: habilidades sociais, consciência social, autoconhecimento e autocontrole. Novamente, para acessar todos os recursos da plataforma é necessário obter um plano pago. Além disso, a plataforma Fit Brains é disponibilizada apenas em inglês. Dessa forma, apenas usuários com conhecimento do idioma podem compreender as instruções para interagir com os jogos ou criar cadastro na plataforma. Para usuários do mundo lusófono, MemoGinga torna-se uma alternativa favorável em função de sua implementação em português.

Todas as ferramentas citadas acima são acessíveis através da Internet. Percebe-se, com isso, que os jogos mencionados dependem de conexão à Internet para serem utilizados. Esse fato restringe o uso das plataformas apenas às pessoas que saibam como utilizar computadores e onde o acesso à rede é disponível. Portanto, no caso de populações isoladas, em condições financeiras limitadas ou sem conhecimento tecnológico suficiente, a utilização desses jogos seria inviável. Nesse aspecto, as características do MemoGinga se sobressaem, pois sua implementação é voltada a televisores fabricados conforme o padrão do Sistema de TV digital Brasileiro à interatividade. 


\section{Jogos e Exercícios Cognitivos}

A qualidade de vida dos idosos apresenta uma correlação com aspectos neuropsicológicos, tais como a cognição. Durante o processo de envelhecimento alguns subsistemas de memória - um dos pilares da cognição - sofrem mais declive que outros. Tais déficits em idosos estão associados à dificuldade na codificação e recuperação de informações [da Costa Pinto 1999]. A preocupação com danos relacionados à memória - devido ao processo natural do envelhecimento - motivou a criação de métodos que estimulassem a cognição. O fato de que idosos que apresentam resultados elevados em avaliações cognitivas [Barnes et al. 2007] têm níveis satisfatórios de autonomia e melhor qualidade de vida também incentiva a exploração deste campo da ciência. Além disso, observou-se que a flexibilidade cognitiva é uma habilidade treinável e, portanto, passível de ser melhorada [Glass et al. 2013].

De acordo com um estudo realizado pela National Institutes of Health (Advanced Cognitive Training for Independent and Vital Elderly), foi descoberto um grande potencial de jogos ofertados a um público de jovens adultos para produzir benefícios cognitivos [Zelinski and Reyes 2009]. Indivíduos que jogam apresentam uma rápida sucessão de estímulos visuais e auditivos aos quais necessitam focalizar sua atenção [Rivero et al. 2012]. Somando-se a isso, existem testes que comprovam melhorias de habilidades cognitivas em resposta ao uso dos jogos, tais como: coordenação mão-olho, dinâmica temporal da atenção sensorial, visão espacial, troca de tarefas, atenção exógena e endógena, entre outros [Latham et al. 2013].

Em relação aos jogadores com idade avançada, outro estudo observou que o grupo treinado com jogos de treinamento cognitivo demonstrou melhora em processamento controlado, atenção e memória imediata e de longo prazo para fotos de familiares em relação aos membros do grupo de controle [Ballesteros et al. 2014]. Além disso, esses jogadores apresentaram níveis superiores em escalas de avaliação de confiança, aceitação social e nível de satisfação com as pessoas ao seu redor em comparação com aqueles que não participaram do treinamento.

\section{MemoGinga}

A ferramenta proposta neste artigo visa a prevenção de problemas cognitivos relacionados a memória, especificamente a de curto prazo. Destarte, o programa usa estratégias de memorização [Santana et al. 2013], empregando o método de categorização. Este método consiste em permitir que informações apresentadas sejam divididas em categorias, exigindo-se assim do usuário a classificação dos dados apresentados como forma de solução dos problemas propostos.

As linguagens utilizadas para o desenvolvimento do programa foram NCL (Nested Context Language) [Soares and Barbosa 2011] e Lua [Ierusalimschy 2006]. NCL é a linguagem declarativa padrão para descrição de conteúdo multimídia do SBTVD, enquanto Lua é a linguagem procedural que pode ser utilizada em conjunto com NCL.

\subsection{Objetivo e Funcionamento do Jogo}

A lógica geral do jogo consiste em exibir figuras geométricas na tela durante uma partida. O jogador deve então comparar uma figura exibida atualmente com a(s) figura(s) exibidas 
anteriormente ao jogador. As comparações devem sempre ser feitas em relação à(s) imagem(ns) exibida(s) imediatamente antes, ou seja, o objeto com que a imagem atual será comparada é alterado a cada nova comparação. Um número fixo de comparações é feito numa partida, variando conforme o nível.

O jogo também varia levemente na forma de comparação de acordo com seus níveis de dificuldade, que são três no total. Em todos os níveis o jogador possui à sua disposição como opões de seleção um botão de confirmação verde, um de negação vermelho e um botão amarelo usado para sair da partida atual.

Cada um dos níveis é implementado como uma aplicação Ginga-NCL diferente. A tela inicial para cada um dos níveis é mostrada na Figura 1. Para esta tela inicial, as opções são de iniciar o jogo ou sair dele. Além disso, uma descrição em áudio do conteúdo escrito na tela inicial também é apresentada nesse momento. Tanto neste menu inicial como durante o jogo, o jogador pode usar as teclas coloridas do controle remoto de seu aparelho de TV para selecionar as opões. Cada botão mostrado no jogo é acionado pela tecla de cor respectiva no controle - sendo verde, vermelho e amarelo as opões usadas ao longo do jogo.

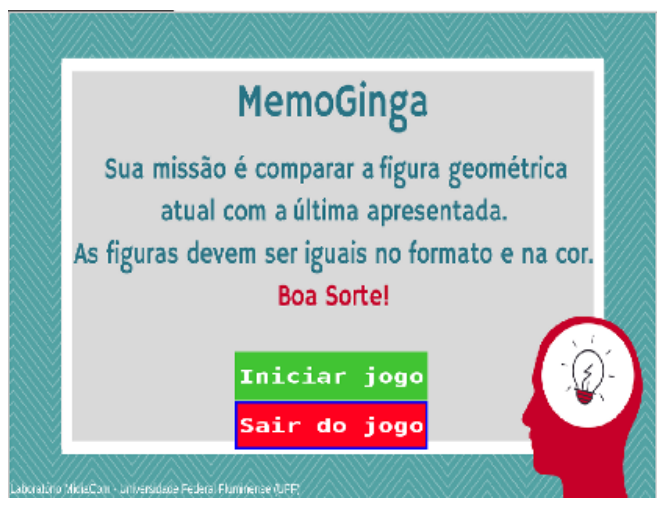

Figura 1. Tela inicial do MemoGinga.

\subsection{Nível 1:}

Neste nível, o jogador deve comparar duas figuras exibidas em sequência e dizer se são iguais ou diferentes. A cada passo do jogo uma figura é exibida para memorização durante um curto período de tempo. Em seguida, uma nova figura é exibida e o jogador deve dizer se a figura atual é a mesma que a anterior. O jogador deve usar o botão de confirmação, caso considere as figuras iguais em forma e cor, ou o botão de negação, caso contrário. Em seguida, uma nova figura para memorização é mostrada e o jogo segue dessa forma. As Figuras 2 e 3 exibem respectivamente a figura inicial e a seguinte exibidas em uma partida.

\subsection{Níveis 2 e 3:}

Os outros dois níveis são extensões do nível 1, seguindo lógica similar de funcionamento. Nesses níveis, a cada rodada um grupo de imagens é exibido e em seguida o jogador deve dizer se uma nova imagem exibida faz parte do grupo de imagens anteriormente mostrado. Ou seja, o jogador deve dizer se uma determinada figura era igual a qualquer 


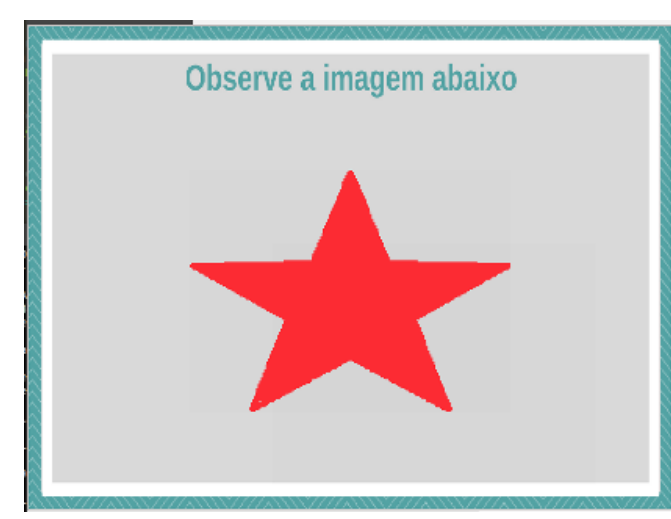

Figura 2. Tela inicial do nível 1 do jogo.

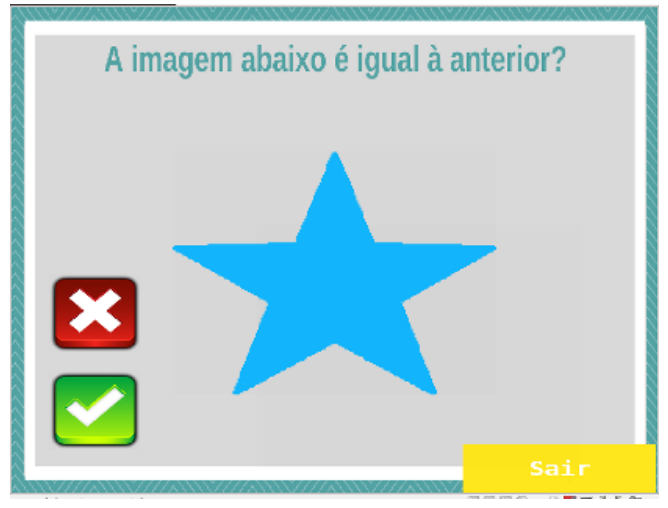

Figura 3. Figura a ser comparada com aquela na tela inicial do nível 1.

outra pertencente ao grupo exibido anteriormente. Os grupos de imagens possuem duas e três figuras para os níveis 2 e 3 respectivamente. Assim como no nível 1, o botão de confirmação indica que a figura única existe no grupo anterior exibido enquanto o botão de negação representa o contrário. Telas do nível 2 são mostradas a seguir pelas Figuras 4 e 5 enquanto o nível 3 é ilustrado pelas Figuras 6 e 7.

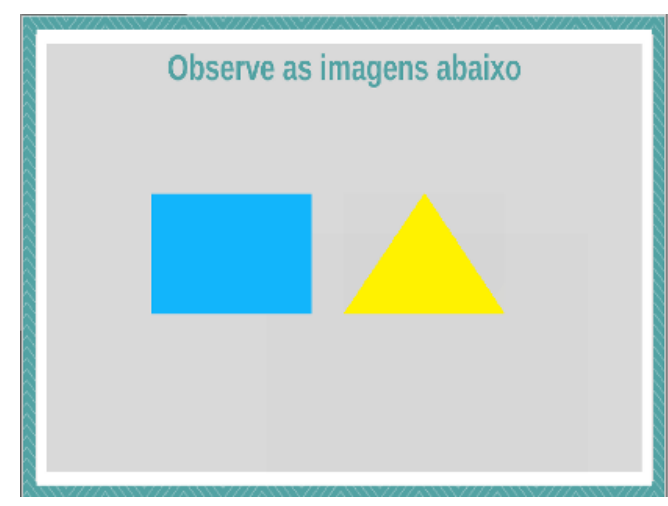

Figura 4. Grupo de figuras no nível 2.

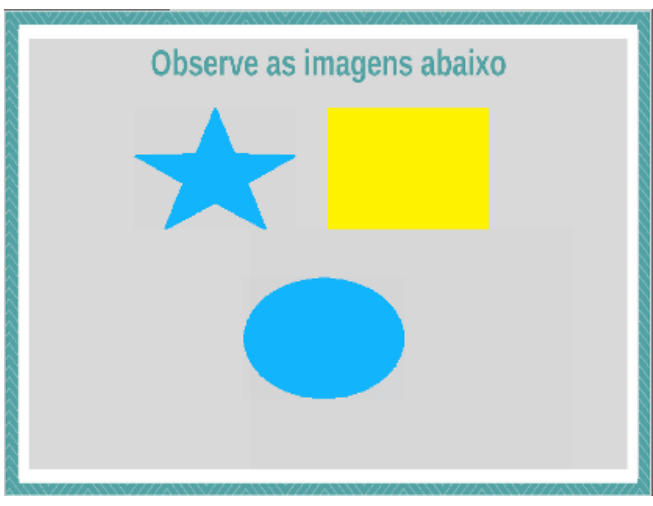

Figura 6. Grupo de figuras no nível 3.

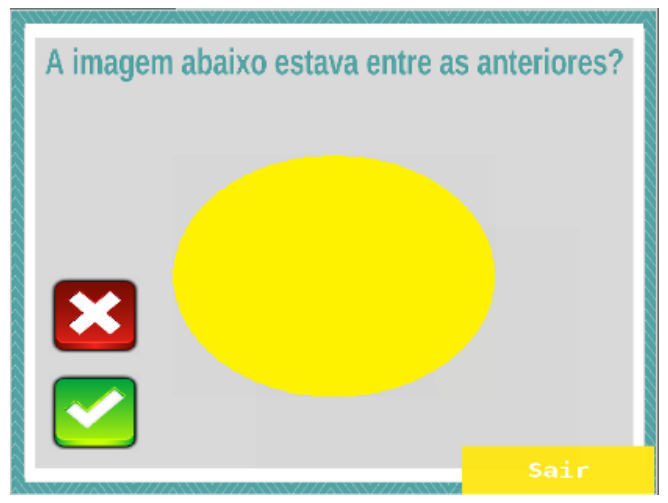

Figura 5. Figura a ser comparada com o grupo no nível 2.

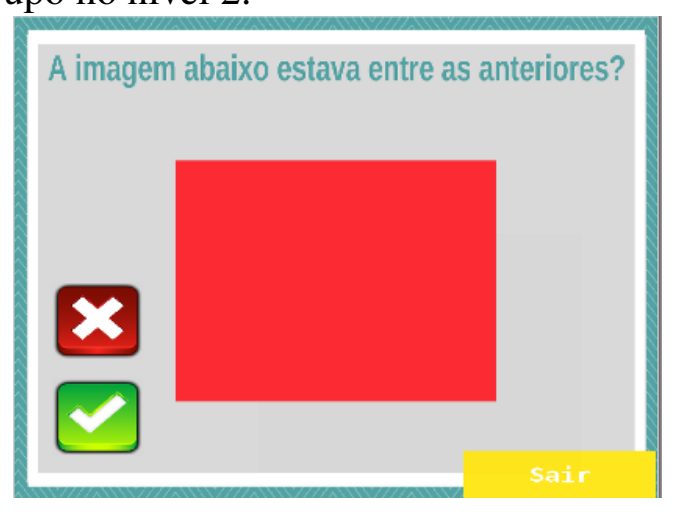

Figura 7. Figura a ser comparada com o grupo no nível 3.

Um número fixo de comparações é efetuado numa partida, sendo dez para o nível 
1 e doze paras os níveis seguintes. O tempo decorrido de cada partida é cronometrado e mostrado junto com a pontuação obtida pelo jogador ao fim de uma partida, como exibe a Figura 8. Assim como a tela inicial, a tela final é a mesma para todos os níveis e após o fim de cada partida retorna-se ao menu inicial.

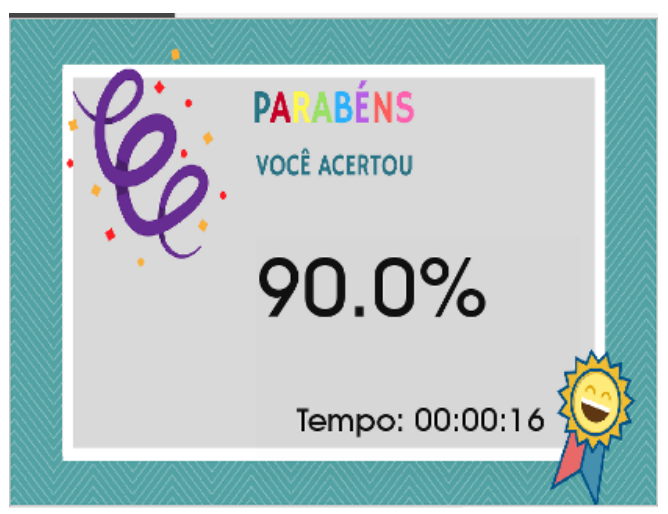

Figura 8. Tela final do MemoGinga.

É possível perceber que a estrutura central do jogo permanece inalterada quando os níveis são adicionados. Isso mostra que a estrutura central pode ser generalizada criando-se um modelo, também chamado de template de composição, que flexibiliza a criação do jogo. Esse template de composição é criado usando a linguagem XTemplate 4.0 [Amorim et al. 2016b], que pode ser utilizada junto a NCL.

A ideia é utilizar o template para descrever a estrutura lógica do jogo de forma genérica, que pode ser especializada conforme o conjunto de mídias utilizado. Essa ideia pode ser visualizada na Figura 9.

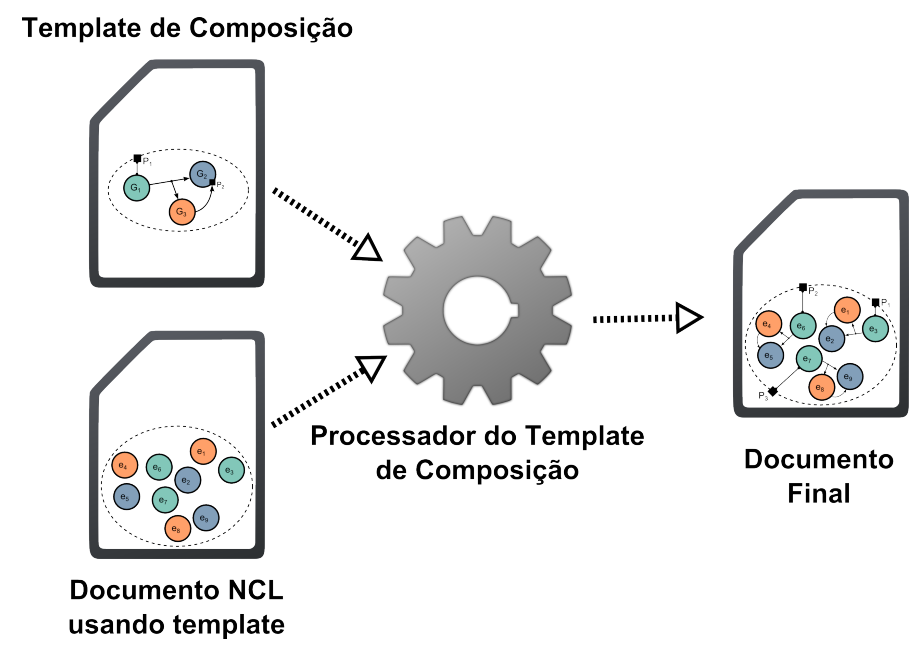

Figura 9. Processamento do Template de Composição

Como mostrado na figura, o template de composição contém todas as relações genéricas necessárias para criar o jogo. $\mathrm{O}$ autor do jogo precisa simplesmente definir as mídias que farão parte do jogo em questão e referenciar o template. Basta então que os dois documentos sejam enviados ao processador do template [Amorim et al. 2016b] para que o código final seja criado. Além da estrutura lógica do jogo, ou seja, as relações 
entre as mídias utilizadas pelo autor, é possível ainda criar um modelo espacial para que o leiaute do jogo também seja descrito de forma genérica, diminuindo o esforço de autoria do game. A linguagem STyLe, apresentada em [Amorim et al. 2016a], pode ser utilizada para tal objetivo.

De acordo com os autores em [Amorim et al. 2016a], a linguagem STyLe fornece alguns elementos espaciais, denominados containers, que são utilizados para gerenciar como um grupo de objetos de mídia será apresentado em uma região espacial. São quatro as possibilidades: gridLayout, flowLayout, stackLayout e carouselLayout.

É possível utilizar dois dos containers oferecidos por STyLe para criar o leiaute espacial do jogo. Os botões de confirmação e negação que estão do lado esquerdo da aplicação podem ser gerenciados pelo container gridLayout com 1 coluna e duas linhas. Já as imagens podem ser gerenciadas através de um container flowLayout com os objetos centralizados na tela. Nesse tipo, as imagens devem ser dispostos dentro do container da esquerda para a direita, linha por linha. Quando não existe mais espaço em uma linha para uma imagem com um determinado tamanho, outra linha é criada abaixo da anterior e o mesmo procedimento é adotado.

A criação desse template espacial facilita a autoria na medida que é possível aumentar o número de imagens na tela sem que haja a necessidade de se criar mais regiões para os novos objetos de mídia.

Outra facilidade que pode ser encontrada com a adição de templates de composição é a possibilidade de produzir o jogo em vários idiomas e deixar que o usuário possa escolher o que lhe convier.

\subsection{Avaliação do MemoGinga}

O primeiro nível de dificuldade do MemoGinga foi testado com pacientes do CRASI (Centro de Referência em Assistência à Saúde do Idoso). O grupo amostral dessa avaliação foi composto de 5 idosos a partir de 70 anos e uma pessoa abaixo dessa faixa etária, conforme apresentado na Tabela 1. Os participantes foram abordados enquanto transitavam no ambiente do CRASI e nenhum deles tinha grandes conhecimentos tecnológicos, especialmente em relação à informática.

Tabela 1. Tabela de dados coletados no teste com pacientes do CRASI.

\begin{tabular}{|l|c|c|c|c|}
\hline Idade (anos) & Escolaridade & Tempo (min) & Acertos (Porcentagem) & Tem Queixas de Memória? \\
\hline 59 & Superior & $01: 42$ & $100 \%$ & Sim \\
\hline 70 & Fundamental (incompleto) & $02: 35$ & $100 \%$ & Sim \\
\hline 78 & Médio (técnico) & $02: 07$ & $80 \%$ & Não \\
\hline 78 & Fundamental & $02: 32$ & $90 \%$ & Sim \\
\hline 82 & Médio (incompleto) & $02: 07$ & $80 \%$ & Não \\
\hline 88 & Fundamental (incompleto) & $02: 40$ & $100 \%$ & Não \\
\hline
\end{tabular}

Após os testes, perguntas sobre a jogabilidade, dificuldade e desconforto ao utilizar o MemoGinga foram realizadas. Nenhum dos participantes queixou-se de dificuldade ou desconforto durante o uso do jogo.

Pode-se notar de acordo com a Tabela 1 que a maioria dos usuários mais idosos obtiveram uma porcentagem de acertos inferior se comparada à dos mais novos. Este 
resultado reafirma a perda de memória em idosos ao longo do tempo observada em outros artigos da literatura [Cancela 2007]. Nota-se ainda que os usuários que levaram mais tempo para concluir o jogo tinham nível de escolaridade inferior.

Posteriormente, testes dos três níveis de dificuldade do MemoGinga foram feitos com outro grupo constituído por 4 idosos, com o propósito de avaliar mais precisamente a usabilidade do sistema. Os membros desse segundo grupo têm a partir de 59 anos de idade. Assim como o grupo anterior, este não tinha prática com aparatos tecnológicos. Os participantes desse segundo teste não estão em avaliação médica relacionada à desordens cognitivas. Alguns dos dados dos participantes encontram-se descritos na Tabela 2. Os dados de tempo e porcentagem de acertos referem-se exclusivamente ao desempenho dos usuários no nível 3. Após jogarem todos os níveis de MemoGinga, os participantes responderam a um questionário centrado na avaliação da usabilidade do sistema, ou seja, analisando aspectos como dificuldade de entendimento da proposta ou funcionamento do jogo, necessidade de aprendizagem para utilização do sistema, complexidade da interface ou jogabilidade, desejo de jogar novamente o MemoGinga e confiança no uso do sistema, entre outros. O questionário segue o padrão do SUS - System Usability Scale - proposto em [Brooke 1996].

A ferramenta de avaliação de usabilidade SUS consiste em um total de dez afirmações relacionadas à usabilidade de um sistema, às quais o participante avaliará numa faixa de 1 (quando discordarem totalmente da afirmação apresentada) a 5 (quando concordarem totalmente com a afirmação) considerando também as possibilidades intermediárias de 2 a 4 (discordo parcialmente, neutro, concordo parcialmente). Um exemplo de afirmação presente no SUS é a seguinte: "eu acho que gostaria de usar esse sistema com frequência", para a qual o participante dará um valor de 1 a 5 que corresponda à sua avaliação sobre a afirmação feita. O SUS é empregado para avaliar a usabilidade de uma ampla gama de produtos, serviços e sistemas propostos e analisados em literatura científica e o mesmo tem sido verificado como um método robusto de análise do referido quesito [Bangor et al. 2008].

Tabela 2. Tabela de dados dos participantes do segundo grupo do teste.

\begin{tabular}{|l|c|c|c|c|c|}
\hline Idade (anos) & Escolaridade & Tempo (min) & Acertos (Porcentagem) & Tem Queixas de Memória? & Pontuação final (SUS) \\
\hline 59 & Fundamental (incompleto) & $01: 16$ & $91,7 \%$ & Sim & 90 \\
\hline 63 & Fundamental (completo) & $04: 30$ & $66,7 \%$ & Sim & 72,5 \\
\hline 75 & Fundamental (completo) & $04: 38$ & $83,3 \%$ & Não & 90 \\
\hline 81 & Fundamental (completo) & $04: 30$ & $75 \%$ & Sim & 65 \\
\hline & & & & & Média (SUS): 79,4 \\
\hline
\end{tabular}

De acordo com os usuários do segundo teste, o jogo apresentou-se de forma clara. Quando questionados se o sistema demonstrou inconsistências, os participantes negaram. Todos afirmaram que outras pessoas não teriam dificuldade em usar o sistema. Além disso, todos os participantes disseram sentir-se confiantes no uso do jogo e que gostariam de jogá-lo mais vezes.

Por outro lado, segundo a tabela, pode-se notar que o participante de idade mais avançada, que declarou não ter queixas de memória (75 anos), teve desempenho em acertos mais próximo àquele registrado pelo participante mais jovem (59 anos). Enquanto isso, com exceção do participante mais jovem, os outros usuários que declararam queixas de memória tiveram desempenho inferior. Nota-se também a significativa diferença de 
tempo de jogo entre o usuário mais jovem e os demais. E por fim, o fato do participante mais jovem ter obtido o melhor desempenho dentre todos reafirma que idosos apresentam dificuldades de memória conforme a idade. Este resultado também foi observado nos testes com o grupo de idosos do CRASI.

Nota-se ainda pela Tabela 2 que, apesar do teste ter sido feito com um pequeno número de usuários, a média SUS acima de 68 [Brooke 1996] indica que o sistema teve um bom resultado no teste de usabilidade. Como trabalhos futuros, mais testes de usabilidade serão feitos com pacientes do CRASI com e sem queixas de memória.

\section{Conclusão}

Por ser uma ferramenta de fácil acesso e que opera sem a necessidade de alta tecnologia, concluímos que MemoGinga pode ser amplamente utilizado para exercício cognitivo de memória curta. Isso é reforçado pelo resultado positivo obtido nos testes de usabilidade feitos com idosos e usuários de outras faixas etárias.

Como trabalhos futuros, almeja-se utilizar o template de composição do jogo para incorporar outros tipos de figuras ou mídias, criando-se assim diferentes tipos de exercícios cognitivos com a mesma lógica geral de funcionamento.

Além disso, ainda usando um template de composição, pretende-se ampliar a gama de idiomas que o MemoGinga disponibiliza ao jogador. Em razão da adoção do middleware Ginga em grande parte dos sistemas de TV digital nos países da América Latina, o idioma espanhol será disponibilizado. O programa também terá como opção de idioma o inglês, assim como as outras plataformas de exercício cognitivo referidas no presente artigo.

Como trabalho futuro, também propõe-se a criação de um banco de dados de informações dos usuários. Isso propiciaria a obtenção de padrões mais específicos dos usuários, possibilitando um estudo mais amplo sobre os efeitos do exercício cognitivo proposto em grupos específicos de faixa etária, gênero, classe social, dentre outros.

Um outro ponto a ser levado em consideração é o refinamento da interação homem-computador tendo em vistas as características linguísticas, psicológica, semiótica entre outras. Em razão disso, tem-se a necessidade de um maior grupo de usuários para o teste de usabilidade.

\section{Agradecimentos}

Os autores agradecem ao CNPq e FAPERJ pelo apoio financeiro ao projeto.

\section{Referências}

Amorim, G. F., dos Santos, J. A. F., and Muchaluat-Saade, D. C. (2016a). Style: Extending $\mathrm{ncl}$ for providing dynamic layouts. In Proceedings of the 22Nd Brazilian Symposium on Multimedia and the Web, Webmedia '16, pages 71-78. ACM.

Amorim, G. F., dos Santos, J. A. F., and Muchaluat-Saade, D. C. (2016b). Xtemplate 4.0: Providing adaptive layouts and nested templates for hypermedia documents. In MultiMedia Modeling: 22nd International Conference, MMM '16, pages 642-653. 
Ballesteros, S., Prieto, A., Mayas, J., Toril, P., Pita, C., de León, L. P., Reales, J. M., and Waterworth, J. (2014). Brain training with non-action video games enhances aspects of cognition in older adults: a randomized controlled trial. Frontiers in Aging Neuroscience, 6:277.

Bangor, A., Kortum, P. T., and Miller, J. T. (2008). An empirical evaluation of the system usability scale. International Journal of Human-Computer Interaction, 24(6):574594.

Barnes, D. E., Cauley, J. A., Lui, L.-Y., Fink, H. A., McCulloch, C., Stone, K. L., and Yaffe, K. (2007). Women who maintain optimal cognitive function into old age. Journal of the American Geriatrics Society, 55(2):259-264.

Brooke, J. (1996). Sus: a quick and dirty usability scale. In Usability Evaluation in Industry. Taylor Francis.

Cancela, D. M. G. (2007). O processo de envelhecimento. http://www.psicologia.pt/artigos/textos/TL0097.pdf. Acessado: 1 mar. 2018.

da Costa Pinto, A. (1999). Problemas de memória nos idosos: Uma revisão. Psicologia, Educação e Cultura, 3:253-295.

Glass, B. D., Maddox, W. T., and Love, B. C. (2013). Real-time strategy game training: Emergence of a cognitive flexibility trait. PLOS ONE, 8:8.

Governo Brasileiro (2014). Levantamento governamental. http://www.brasil.gov.br/governo/2014/12/televisao-ainda-e-o-meio-de-comunicacaopredominante-entre-os-brasileiros. Acessado: 18 jan. 2018.

Ierusalimschy, R. (2006). Programming in Lua, Second Edition. Lua.Org.

Ishihara, K., Nagamachi, M., Kohchi, M., and Ishihara, S. (2012). Design requirements for a cognitive training game for elderly or challenged people. Kansei Engineering International Journal, 11(4):241-246.

Latham, A. J., Patston, L. L. M., and Tippett, L. J. (2013). The virtual brain: 30 years of video-game play and cognitive abilities. Frontiers in Psychology, 4:629.

Lumos Labs, Inc (2014). Lumosity. https://www.lumosity.com/. Acessado: 18 jan. 2018.

Matos, L. F., Santos, M. H., Boechat, Y., and Muchaluat-Saade, D. C. (2018). MemoGinga: Exercício Cognitivo para Idosos Utilizando o Sistema Brasileiro de TV Digital Interativa. Anais da VI Escola Regional de Computação Aplicada à Saúde, Niterói, RJ.

NBR 15606-2:2011 (2011). Televisão digital terrestre - codificação de dados e especificações de transmissão para radiofusão digital parte 2: Ginga-ncl para receptores fixos e móveis - linguagem de aplicação xml para codificação de aplicações. Standard, Associação Brasileira de Normas Técnicas.

Nouchi, R., Taki, Y., e Hiroshi Hashizume, H. T., Akitsuki, Y., Shigemune, Y., Sekiguchi, A., Kotozaki, Y., Tsukiura, T., Yomogida, Y., and Kawashima, R. (2012). Brain training game improves executive functions and processing speed in the elderly: A randomized controlled trial. PLOS ONE, 7(1):1-9. 
Oei, A. C. and Patterson, M. D. (2013). Enhancing cognition with video games: A multiple game training study. PLOS ONE, 8:1-16.

Posit Science (2016). BrainHQ. https://www.brainhq.com/. Acessado: 18 jan. 2018.

Rivero, T. S., Querino, E. H. G., and Starling-Alves, I. (2012). Videogame: seu impacto na atenção, percepção e funções executivas. Revista Neuropsicologia Latinoamericana, $4(3): 38-52$.

Rosetta Stone (2018). Fit Brains. http://www.fitbrains.com/. Acessado: 10 mar. 2018.

Santana, F., Lima, T. B., Bettine, E., and Moreira, E. (2013). Estimulação Cognitiva Para Idosos - Ênfase em Memória. Editora Atheneu.

Soares, L. F. G. and Barbosa, S. D. J. (2011). Programando em NCL 3.0, 2a. Edição, Versão 2.1. Elsevier Campos.

Zelinski, E. M. and Reyes, R. (2009). Cognitive benefits of computer games for older adults. Gerontechnology: International Journal on the Fundamental Aspects of Technology to Serve the Ageing Society, 8(4):220-235. 